\title{
A Comparative Study of Clinical Intervention and Interventional Photothermal Therapy for Pancreatic Cancer
}

\author{
Yanyan Hu, Chongwei Chi, Shunhao Wang, Lingxiong Wang, Ping Liang, Fangyi Liu, \\ Wenting Shang, Weiwei Wang, Fengrong Zhang, Shanshan Li, Heyun Shen, Xiaoling Yu,* \\ Huipu Liu,* and Jie Tian*
}

\begin{abstract}
Although nanoparticle-based photothermal therapy (PTT) has been intensively investigated recently, its comparative efficiency with any clinical cancer treatments has been rarely explored. Herein for the first time we report a systematic comparative study of clinical iodine-125 ( ${ }^{125}$ I) interstitial brachytherapy (IBT-125-I) and interventional PTT (IPTT) in an orthotopic xenograft model of human pancreatic cancer. IPTT, based on the nanoparticles composing of anti-urokinase plasminogen activator receptor (UPAR) antibody, polyethylene glycol (PEC), and indocyanine green (ICG) modified gold nanoshells (hereinafter uIGNs), is directly applied to local pancreatic tumor deep in the abdomen. In comparison to IBT-125-I, a 25\% higher median survival rate of IPTT with complete ablation by one-time intervention has been achieved. The IPTT could also inhibit pancreatic tumor metastasis which can be harnessed for effective cancer immunotherapy. All results show that this IPTT is a safe and radical treatment for eradicating tumor cells, and may benefit future clinical pancreatic cancer patients.
\end{abstract}

Pancreatic cancer (PC) is one of the most lethal malignancies of humans, with a five-year survival rate of less than $5 \%{ }^{[1]}$ Although radical surgery is currently the first treatment selected for PC, approximately $80 \%$ of patients present at a stage not manageable with curative surgery. ${ }^{[1 a, 2]}$ Iodine- 125
( ${ }^{125}$ I) interstitial brachytherapy (IBT-125-I), as a form of radiotherapy, provides a new and potential choice for the treatment of unresectable PC patients. In this procedure, ${ }^{125} \mathrm{I}$ radioactive seeds are permanently implanted into the tumor or region of interest, thereby avoiding side effects in surrounding normal tissues. ${ }^{[3]}$ However, even after IBT-125-I, some patients still suffer from high recurrence rates, possibly owing to (1) inaccurate delineation of tumor margins; (2) uneven dose distribution, yielding incomplete radioactive dose coverage of the tumors; or (3) failed local control of the whole tumor as a result of the long therapeutic window of ${ }^{125}$ I seeds, particularly when the tumor doubling time is shorter. Thus, there remains a demand for better treatment options.

To achieve superior therapeutic effects against PC, multiple therapies and platforms have recently been developed, such as photothermal, ${ }^{[4]}$ photodynamic, ${ }^{[5]}$ sonodynamic, ${ }^{[6]}$ and immune therapies. ${ }^{[7]}$ Of these, nanomaterials-based photothermal therapy (PTT), which can eradicate tumors by rapidly converting near-infrared (NIR) light energy into ablative heat via electron-phonon and
Dr. Y. Y. Hu, Prof. P. Liang, Dr. F. Y. Liu, Prof. X. L. Yu

Department of Interventional Ultrasound

General Hospital of People's Liberation Army

Beijing 100853, China

E-mail: dyux1301@aliyun.com

Dr. Y. Y. Hu, Dr. C. W. Chi, Dr. W. T. Shang, Prof. J. Tian

Key Laboratory of Molecular Imaging

Institute of Automation

Chinese Academy of Sciences

Beijing 100190, China

E-mail: jie.tian@ia.ac.cn

Dr. S. H. Wang, Dr. W. W. Wang, Dr. F. R. Zhang, Dr. S. S. Li,

Prof. H. Y. Shen, Prof. H. Y. Liu

Bionanomaterials \& Translational Engineering Laboratory

Beijing Key Laboratory of Bioprocess

State Key Laboratory of Chemical Resource Engineering

Beijing Laboratory of Biomedical Materials

Beijing University of Chemical Technology

Beijing 100029, China

E-mail: liuhy@mail.buct.edu.cn
Dr. S. H. Wang

State Key Laboratory of Environmental Chemistry and Ecotoxicology

Research Center for Eco-Environmental Sciences

Chinese Academy of Sciences

Beijing 100085, China

Dr. S. H. Wang, Prof. J. Tian

University of Chinese Academy of Sciences

Beijing 100049, China

Dr. L. X. Wang

Cancer Center lab

Division of Internal Medicine

General Hospital of People's Liberation Army

Beijing 100853, China

DOI: 10.1002/adma.201700448 
phonon-phonon coupling interactions, ${ }^{[8]}$ has gained remarkable interest. In particular, AuroShell particles (Nanospectra Biosciences Inc., Texas), a type of gold nanoshells (GNs), were recently approved for evaluation in a clinical trial in patients with refractory and/or recurrent tumors of the head and neck (ClinicalTrials.gov Identifier: NCT00848042). Moreover, our previous report also presented that gold nanoshells exhibit good efficacies against cancer cells. ${ }^{[9]}$ However, this therapy modality is restricted by several limitations, including a limited light penetration depth for deep-buried tumors, the possibility of damaging normal tissues, and compromised therapeutic effects by threshold laser power for skin tolerance. ${ }^{[10]}$ Given these issues, we hypothesize that a loco-regional interventional method for delivery of PTT might comprise a good choice for clinical practice, especially for PC patients with deep-buried tumors in the abdomen. Surprisingly, to date, no studies have evaluated the efficacy of interventional PTT (IPTT) compared with other clinical intervention therapy modalities, impelling a detailed investigation.

Herein we developed an IPTT modality to kill PC cells deep in the addomen. The IPTT device was self-developed by running an NIR optical fiber through an 18-gauge (G) percutaneous transhepatic cholangiography (PTC) needle, enabling access to deep PC tissues (Figure S1, Supporting Information). The anti-urokinase plasminogen activator receptor (uPAR) antibody, polyethylene glycol (PEG), and indocyanine green (ICG) conjugated GNs (herein after uIGNs) were chosen as the PTT agent (Scheme 1). ICG, the first Food and Drug Administration (FDA)-approved NIR fluorescent dye, was included in the nanoparticle design for its fluorescence imaging capabilities and the added photothermal effects it afforded. Moreover, we conducted, to the best of our knowledge, the first systematic comparison of clinical IBT-125-I and IPTT in an orthotopic xenograft model of human pancreatic cancer (Scheme 1). The IPTT modality described herein provides the following specific advantages: (i) GNs exhibit large absorption cross-sections in NIR region, good photostability, easily conjugation of biomolecules, and excellent biocompatibility for PTT and multi-modal imaging, ${ }^{[9 a, c, 11]}$ (ii) the anti-uPAR antibody (uPAR Ab) has shown exceptional promise in active targeting, ${ }^{[12]}$ particularly for deep penetration of PC with dense dysplastic stroma; ${ }^{[13]}$ (iii) the aforementioned uIGNs could be used to both visualize tumors (via CT and optical imaging) and mediate PTT for PC. The results demonstrate that in comparison to IBT-125-I, a $25 \%$ higher median survival rate of IPTT and complete tumor ablation by one-time intervention are obtained in this study. Further, uIGNs exhibited excellent CT and NIR imaging, allowing real-time monitoring of therapeutic processes and tumor metastases of less than $2 \mathrm{~mm}$. Additionally, the IPTT modality could also inhibit pancreatic tumor metastasis in mice. We therefore believe that the result of our study will have a significant impact on future clinical translation of nanomaterialsbased IPTT for patients with unresectable pancreatic tumor or tumor metastases.

GNs, comprised of a mesoporous silica nanorattle core and a thin gold nanoshell, were synthesized in an aqueous solution via a seed-growth procedure, as previously described. ${ }^{[9]}$ The resulting GNs had an average diameter of $\approx 155 \mathrm{~nm}$ (Figure 1a and Figure S2a,b, Supporting Information) with well-defined spherical morphology and high uniformity, which were suitable

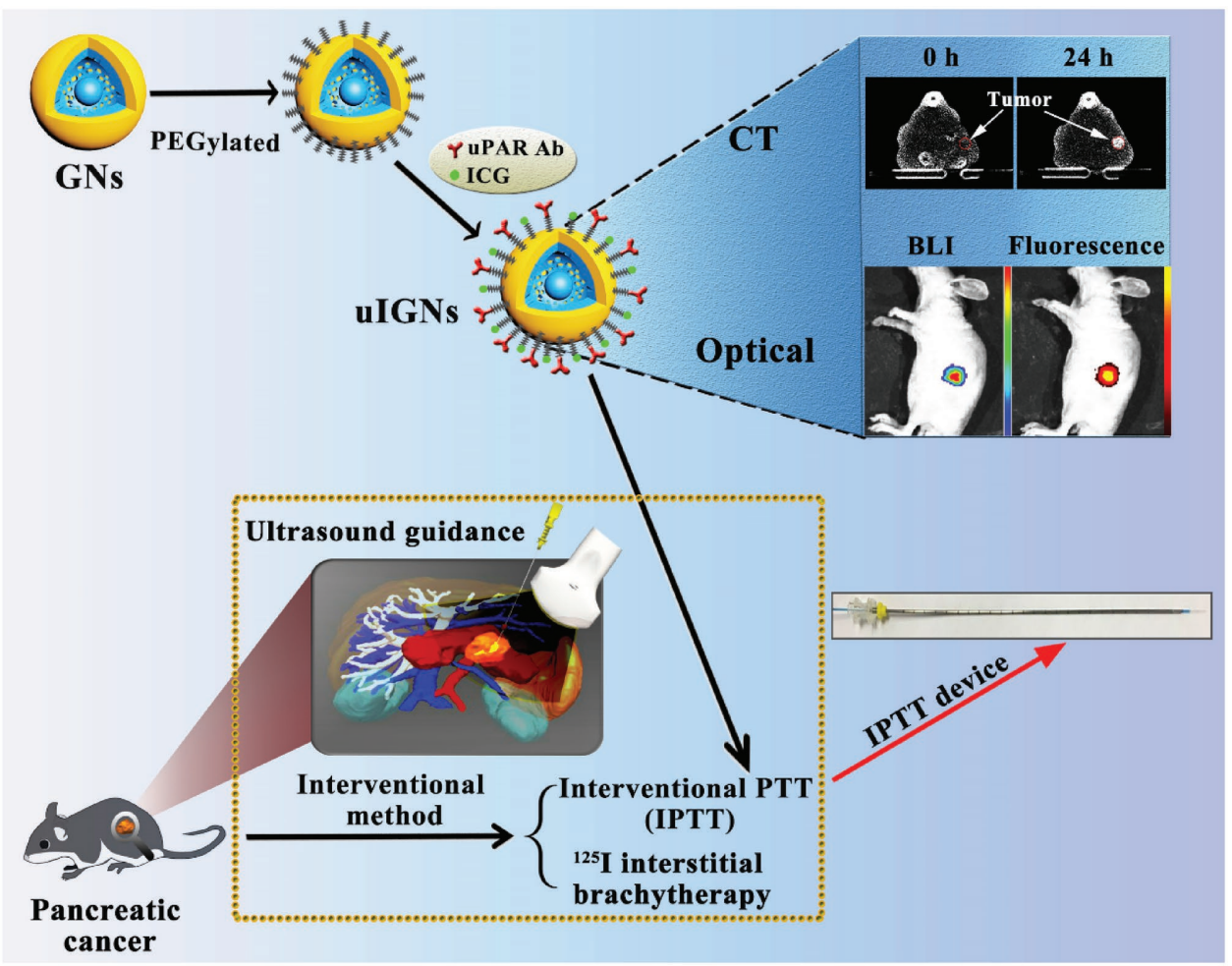

Scheme 1. Schematic illustration of the comparative study of interventional photothermal therapy and ${ }^{125}$ I interstitial brachytherapy. BLI: bioluminescence imaging. 

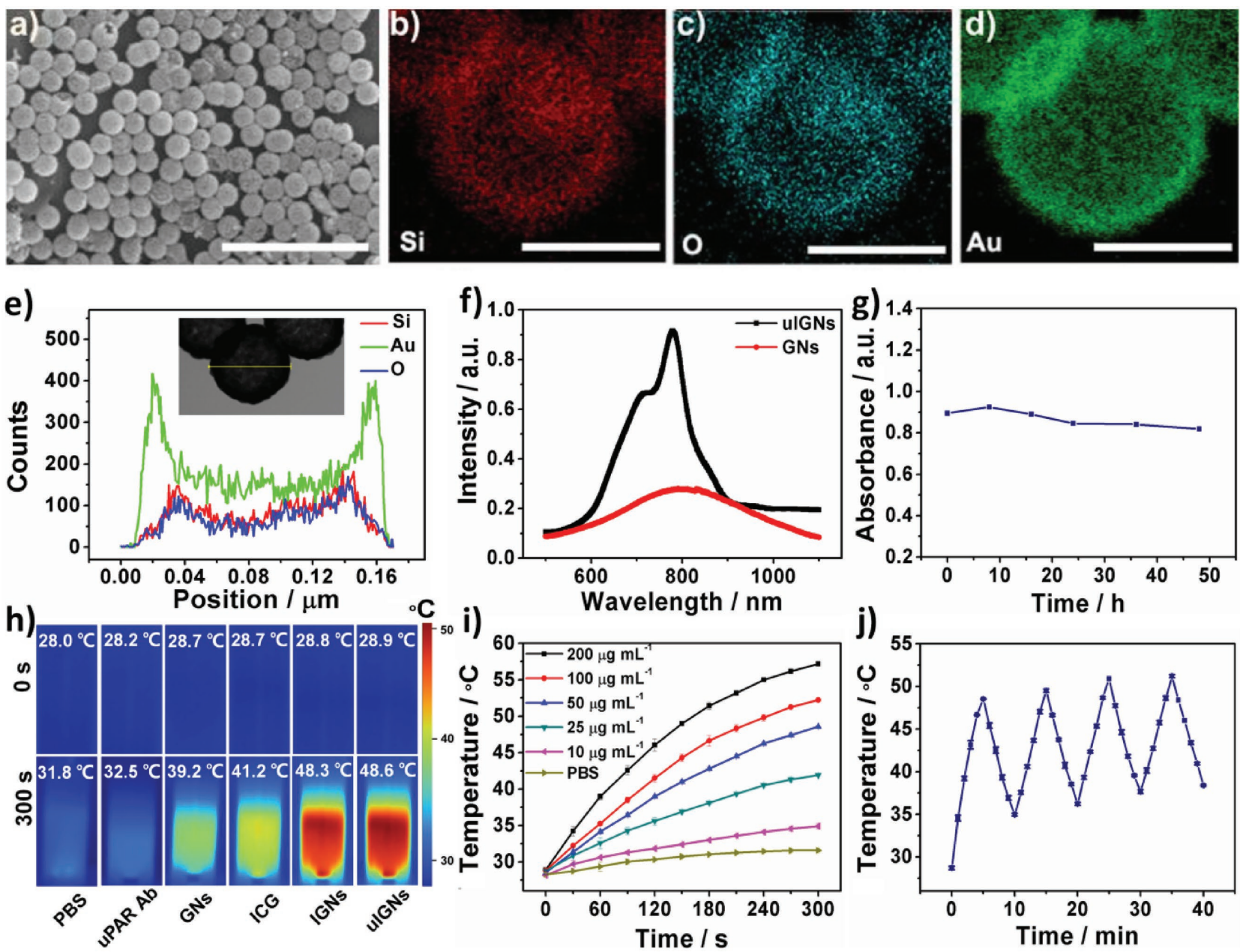

Figure 1. Synthesis and characterization. a) SEM image of GNs. Scale bar is $1 \mu \mathrm{m}$. Element mapping for b) Si, c) O, and d) Au of GNs. Scale bar is $100 \mathrm{~nm}$. e) Cross-sectional compositional line profiles of GNs. f) UV-vis-NIR absorption spectrum of GNs and ulGNs. g) Serum stability of uIGNs at a concentration of $50 \mu \mathrm{g} \mathrm{mL} \mathrm{m}^{-1}$ observed for $48 \mathrm{~h}$. h) NIR thermal imaging before and after NIR irradiation. i) Photothermal effects of uIGNs aqueous dispersions at different concentration $\left(0,10,25,50,100\right.$, and $\left.200 \mu \mathrm{g} \mathrm{mL}^{-1}\right)$ under NIR laser irradiation $\left(\lambda=808 \mathrm{~nm} ; 2.0 \mathrm{~W} \mathrm{~cm}{ }^{-2}\right)$ for $300 \mathrm{~s}$. j) Real-time temperature measurement of uIGNs aqueous dispersion $\left(50 \mu \mathrm{g} \mathrm{mL}^{-1}, 1 \mathrm{~mL}\right)$ under $808 \mathrm{~nm} \mathrm{NIR} \mathrm{light} \mathrm{irradiation} \mathrm{with} \mathrm{a} \mathrm{power} \mathrm{density} \mathrm{of} 2.0 \mathrm{~W} \mathrm{~cm}^{-2}$, for four cycles. Each cycle consisted of 5-min irradiation followed by a 5-min cooling phase. SEM: Scanning electron microscopy.

for blood circulation and cellular uptake due to the enhanced permeability and retention (EPR) effect of solid tumors. Element mapping of GNs (Figure 1b-d) not only showed direct evidence of the rattle structure of silica nanorattles (Figure 1b,c), but also demonstrated the uniform coating of gold nanoshells over these nanorattles (Figure 1d). These results were also supported by a line-profile analysis using the high-angle annular dark-field scanning transmission electron microscopy-energy-dispersive X-ray images (Figure 1e). PEG, ICG, and uPAR Ab were conjugated to GNs following a previously described method, for the functionalization of long blood circulation time, ${ }^{[9 b]}$ NIR fluorescence, and high-affinity active targeting. The conjugation efficiency of ICG was $27.3 \mathrm{wt} \%$. Unless noted otherwise, the amount of free ICG used in the experiments was equal to that conjugated to uIGNs. The emission peak of uIGNs was located at $817 \mathrm{~nm}$ (Figure S2c, Supporting Information), consistent with the spectra of free ICG, while the absorption peak covered the NIR region (Figure 1f), consistent with that of GNs and ICG. ${ }^{[14]}$ These data indicated that UIGNs maintained their unique optical properties after modification. Furthermore, the absorption peaks remained without aggregation or precipitation during incubation in serum for $48 \mathrm{~h}$ (Figure $1 \mathrm{~g}$ ), suggesting excellent stability. To confirm the photothermal effect of uIGNs, an NIR thermographic system was utilized to measure the temperature changes of all samples $\left(50 \mu \mathrm{g} \mathrm{mL}^{-1}\right)$ before and after NIR light irradiation (808 nm, $\left.2.0 \mathrm{~W} \mathrm{~cm}{ }^{-2}, 300 \mathrm{~s}\right)$. Compared with phosphate-buffered saline (PBS), uPAR Ab, GNs, and free ICG, the temperature of the sample containing uIGNs and ICG-GNs (hereinafter IGNs) increased dramatically from $\approx 28$ to $\approx 48{ }^{\circ} \mathrm{C}$ (Figure $1 \mathrm{~h}$ and Figure S2d, Supporting Information) in $300 \mathrm{~s}$ because of the additive effect of having both ICG and GNs present in the samples. Meanwhile, the increase of temperature was in a time-dependent and concentrationdependent manner as indicated in Figure 1i. Further cycle heating experiments $(5 \mathrm{~min}$ irradiation followed by a $5 \mathrm{~min}$ cooling period) were carried out, suggesting that repeated NIR light irradiation did not significantly affect the photothermal performance of uIGNs (Figure 1j). 

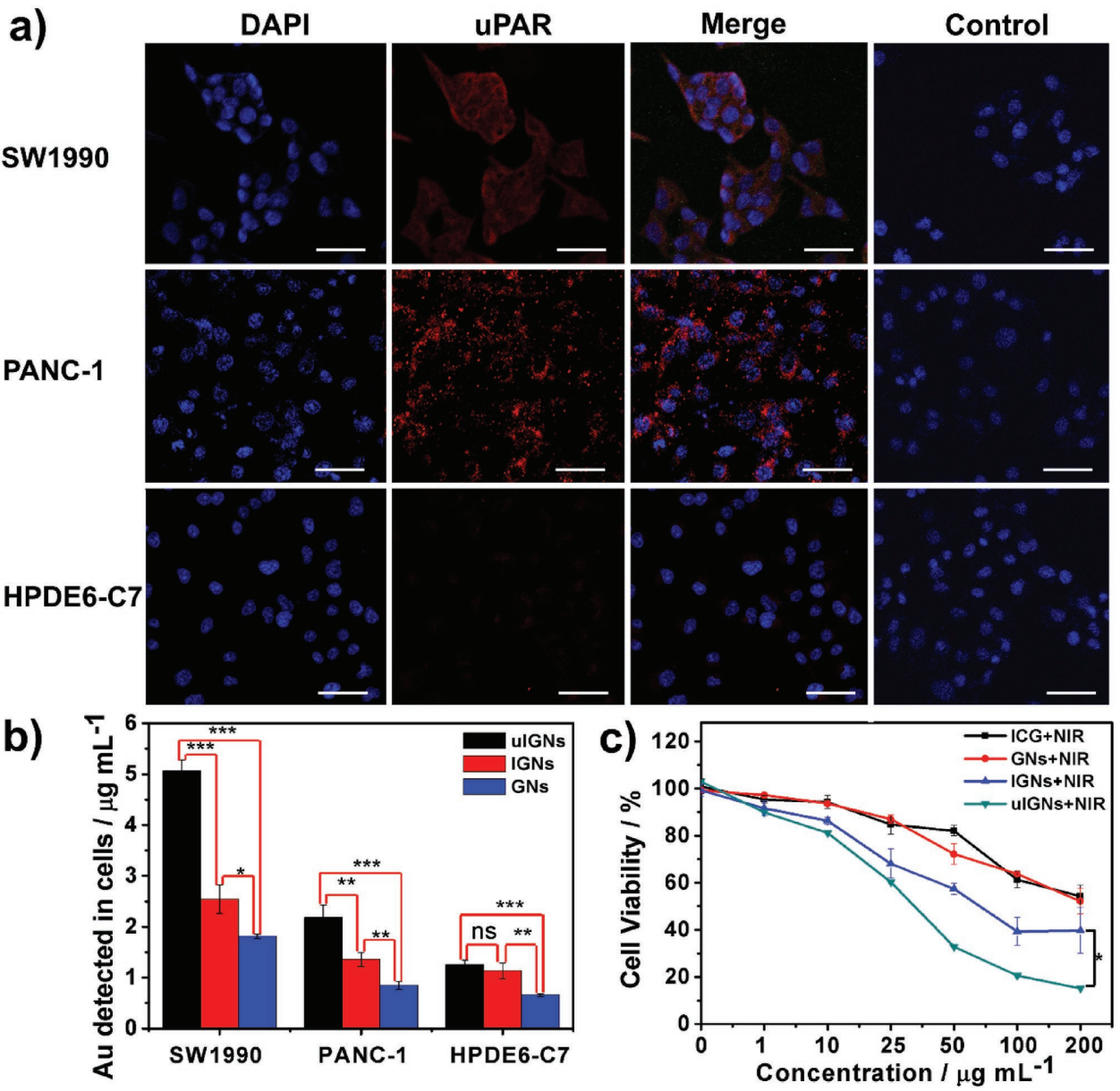

Figure 2. Evaluation of uPAR expression, in vitro cellular uptake and cytotoxicity. a) uPAR expression is relatively high in SW1990 cells and PANC-1 cells, but low in normal human pancreatic ductal epithelial cells HPDE6-C7 by immunofluorescence labeling. Controls in each cell line were only incubated with secondary antibody. The merged images of control groups were listed right. Scale bar is $50 \mu \mathrm{m}$. b) SW1990, PANC-1, and HPDE6-C7 cells were preincubated with uIGNs, IGNs, and GNs (at $50 \mu \mathrm{g} \mathrm{mL} \mathrm{m}^{-1}$ ) for $24 \mathrm{~h}$. ICP-MS was performed to detect Au element entered in SW1990, PANC-1 and HPDE6C7 cells. c) The viability of SW1990 cells incubated with uIGNs, IGNs, ICG, and GNs at different concentrations for $24 \mathrm{~h}$ and treated with NIR laser irradiation $\left(\lambda=808 \mathrm{~nm} ; 2.0 \mathrm{~W} \mathrm{~cm}^{-2}\right)$ for $5 \mathrm{~min}$. Data represent mean \pm SD of triplicate experiments. $\mathrm{ns}: P>0.05 ; * P<0.05 ; * * P<0.01 ; * * * * P<0.001$.

To validate whether uPAR could be used as a specific pancreatic biomarker, we analyzed uPAR expression from three levels, including in vitro cell lines, a mouse model of human PC, as well as surgical specimens from patients. The human PC cell lines PANC-1 and SW1990, and the normal human pancreatic duct epithelial cell line HPDE6-C7 were used. As shown in Figure 2a, SW1990 cells and PANC-1 cells exhibited elevated UPAR expression, while HPDE6-C7 cells exhibited low levels of UPAR expression. Likewise, in the SW1990 orthotopic pancreatic tumor model with high metastatic potential, strong and widely distributed expression of uPAR was observed in primary and metastatic tumor tissues, whereas normal tissues exhibited extremely low UPAR expression, as determined via immunohistochemistry (IHC) (Figure S3, Supporting Information). Lastly, the expression of UPAR in 43 surgical specimens from patients with PC $(n=15)$ and pancreas cystic neoplasms (PCN; $n=28)$ via IHC was investigated. While uPAR was expressed at high levels in PC tissues and in borderline tumor tissues with high malignant potential, e.g., intraductal papillary mucinous neoplasm II (IPMN-II), this factor was expressed at low levels or not at all in benign PCN tissues and normal pancreatic tissues (Figure S4, Supporting Information). Together, these findings indicate that UPAR is selectively and strongly involved in pancreatic cancer, and can be used as a reliable biological target for live imaging or targeted therapy.

Targeting ability and biocompatibility are two major obstacles in the development of targeted nanotheranostic agents, which can precisely target cancer cells, increase cellular uptake and deliver antitumor therapies. ${ }^{[15]}$ As shown in Figures S5 and S6 (Supporting Information), transmission electron microscopy (TEM) analysis of different cell lines confirmed the intracellular uptake of IGNs and UIGNs. To quantitatively evaluate the celltargeting capabilities and cellular uptake of UIGNs, IGNs, and GNs, SW1990, PANC-1, and HPDE6-C7 cells were preincubated with each of these individual particles $\left(50 \mu \mathrm{g} \mathrm{mL}^{-1}\right)$ and subjected to inductively coupled mass spectrometry (ICP-MS) analysis to detect gold $(\mathrm{Au})$ content inside the cells. Compared with the IGN and GN groups, a significantly higher quantity of Au was detected in the uPAR-overexpressing SW1990 and PANC-1 tumor cells in the uIGN group, suggesting that a 
significantly higher amount of uIGNs with active targeting ability entered these cells. Conversely, in HPDE6-C7 cells, which exhibited low uPAR expression, the amount of Au uptake was nearly identical in the IGN and uIGN groups $(P>0.05)$ (Figure 2b). To further evaluate the cellular uptake of uIGNs, IGNs, and free ICG, the fluorescence intensity of ICG (an indication of the respective amount of different nanoparticles entering tumor cells) was evaluated in SW1990, PANC-1, and HPDE6-C7 cells. Compared with IGNs, a significantly higher quantity of uIGNs was observed in uPAR-overexpressing SW1990 and PANC-1 tumor cells, while nearly the same quantity of uIGNs and IGNs entered HPDE6-C7 cells (Figure S7, Supporting Information). These results suggest that uIGNs can effectively and specifically target uPAR-overexpressing tumor cells and yield increased cellular uptake. It is also interesting to note that the cellular uptake of IGNs was higher than that of free ICG in all three cell lines, regardless of uPAR expression (Figure S7, Supporting Information). This could be due to the fact that, compared with free ICG, IGNs exhibit a greater capacity to be endocytosed, thereby allowing for greater cellular uptake of ICG per unit of time. ${ }^{[14]}$

To evaluate the biocompatibility and cytotoxicity of uIGNs, IGNs, ICG, and GNs for biomedical applications, the viability of SW1990 and PANC-1 cells, preincubated with different concentrations of each sample, was determined using Cell Counting Kit-8 (CCK-8) assay. No obvious cytotoxicity was observed in these cells after $24 \mathrm{~h}$ incubation with any of the respective nanoparticles at $37^{\circ} \mathrm{C}$ (Figure S8a,b, Supporting Information), suggesting that these nanoparticles are safe and nontoxic to cells when used alone. Subsequently, the phototoxicity effects of the samples were examined using CCK-8 assay. Under NIR irradiation, concentration-dependent cytotoxicity profile was verified in both SW1990 and PANC-1 cells (Figure 2c and Figure S8c, Supporting Information). The viabilities of NIRirradiated cells treated with uIGNs were significantly different from those treated with IGNs $(P<0.05)$ (Figure 2c and Figure S8c, Supporting Information). The half-maximum inhibiting concentrations ( $\mathrm{IC}_{50}$ value) for the uIGN group were 32.2 and $33.7 \mu \mathrm{g} \mathrm{mL}^{-1}$ for SW1990 and PANC-1 cells, respectively, which was $59.1 \%$ and $49.3 \%$ lower than that of the IGN group ( $\mathrm{IC}_{50}$ value: 77.81 and $66.55 \mu \mathrm{g} \mathrm{mL} \mathrm{m}^{-1}$, respectively), as shown in Figure S8d (Supporting Information). These results illustrate that the uptake efficiency and photothermal cytotoxicity of uIGNs are significantly higher than those of the non-targeted nanoparticles, presumably owing to the active targeting ability of uIGNs for uPAR-overexpressing tumor cells.

To investigate the in vivo targeting ability and dynamic biodistribution of the different types of nanoparticles, SW1990 tumor-bearing mice were divided into three groups and injected with uIGNs, IGNs, and ICG via the tail vein, respectively. An in vivo imaging system (IVIS) Spectrum was then used to trace the distribution of the probe, and a series of images were collected at different time points post-injection. As shown in Figure 3a,b, uIGNs accumulated in both primary and metastatic tumor lesions. In vivo behavior of the synthesized nanoparticles was investigated using ICP-MS analysis as well. As shown in Figure S9a-d (Supporting Information), the nanoparticles showed both liver and spleen uptakes, implying that UIGNs and IGNs could accumulate in the organs of the reticuloendothelial system. To compare the tumor-targeting specificity of uIGNs and IGNs, tumor-to-normal tissue contrast ratios $(\mathrm{T} / \mathrm{N})$ based on ex vivo images were calculated and ICP-MS analysis of gold level in tumor tissues was performed. At 18-24 $\mathrm{h}$ post-injection, the accumulation amount of UIGNs within tumors gradually reached a peak, which was significantly higher than that of IGNs $(P<0.05)$ (Figure $3 \mathrm{C}$ and Figure S10a, Supporting Information). Meanwhile, as depicted in Figure S10b (Supporting Information), the quantity of nanoparticles based on ex vivo images that accumulated in tumors in the uIGN group was significantly higher than that in the IGN group $(P<0.01)$ and the ICG group $(P<0.001)$. Remarkably, in vivo optical fluorescence imaging was even sensitive enough to detect extremely small $(<2 \mathrm{~mm}$ in diameter) tumor metastases after injection of uIGNs (Figure 3d,e). All the results reveal that the targeting effect of $\mathrm{uPAR} A \mathrm{~b}$ can dramatically change the distribution of uIGNs, thereby increasing total tumor uptake in both primary and metastatic tumor lesions to a greater extent than the EPR effect alone.

The use of gold nanostructures as CT contrast agents has received considerable attention for their good biocompatibility and high levels of X-Ray absorption. ${ }^{[16]}$ The CT imaging performance of uIGNs was evaluated in SW1990 tumor-bearing mice. Analysis of CT images taken before and at $24 \mathrm{~h}$ post-injection of UIGNs into the tail vein showed a significant enhancement in the pancreatic tumor site (Figure S11a, Supporting Information), indicating that theranostic uIGNs show promise for dualmodality (CT and optical imaging)-based diagnosis and can accurately delineate tumor margins.

Given the observed differences in the in vivo biodistribution of UIGNs, IGNs, and ICG, it is necessary to explore the in vivo therapeutic effects of IPTT. Therefore, for the first time, we performed a comparative analysis of IPTT and the clinical intervention, IBT-125-I. SW1990 tumor-bearing mice were randomly divided into five groups ( $n=8$ per group): mice receiving $\mathrm{uIGNs}$ with NIR laser treatment (Group A), IGNs with NIR laser treatment (Group B), PBS with NIR laser treatment (Group C), or IBT-125-I (Group D), as well as a control group (Group E). According to the results of the biodistribution study, we decided to deliver IPTT at $24 \mathrm{~h}$ post-injection (Figure 4a). As shown in Figure $4 \mathrm{~b}$, a rapid rise in surface temperature was observed in the tumor regions of the mice in Group A and B, and these temperatures were sufficient for tumor ablation. In contrast, temperatures in the PBS/laser-treated tumor region (Group C) remained below $40{ }^{\circ} \mathrm{C}$ throughout the procedure. These results confirm the effective in vivo photothermal effect of uIGNs and IGNs. Subsequently, we made a preoperative treatment planning for IBT-125-I using the UIGN-enhanced CT images, where one ${ }^{125}$ I seed was needed for each mouse (Figure S11b, Supporting Information). The procedure was guided by ultrasound to ensure that the seed was implanted in the middle of the tumor (Figure S11c, Supporting Information).

To evaluate the potential therapeutic effects of these strategies, bioluminescence imaging (BLI) was utilized to continuously monitor each treated lesion at $3 \mathrm{~d}$ intervals. Notably, while the tumors in Group A were completely ablated within $15 \mathrm{~d}$, residual tumors were observed in Groups B, C, and D following treatment (Figure 5a). The one-time treatment success rates (i.e., no residual tumor detected post-treatment) for the five 
a)

BLI

Fluorescence (uIGNs)

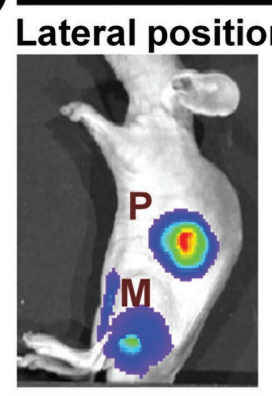

Supine position

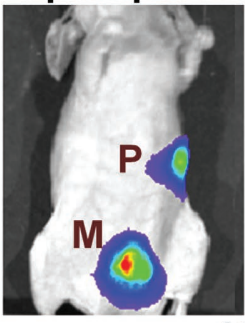

b)

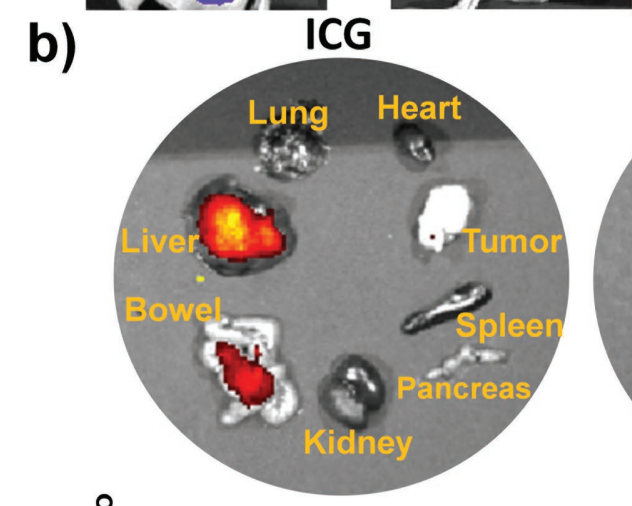

c)
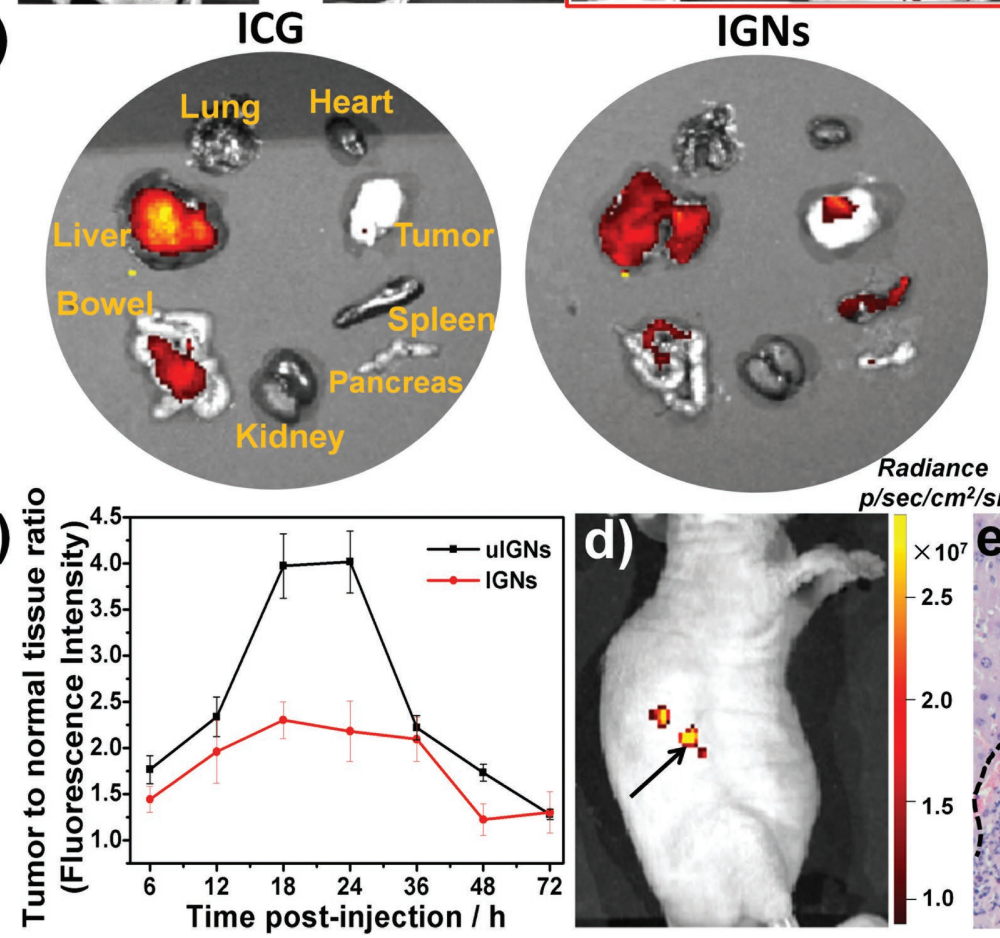

$3 \mathrm{~h}$

$6 \mathrm{~h}$

Radiance
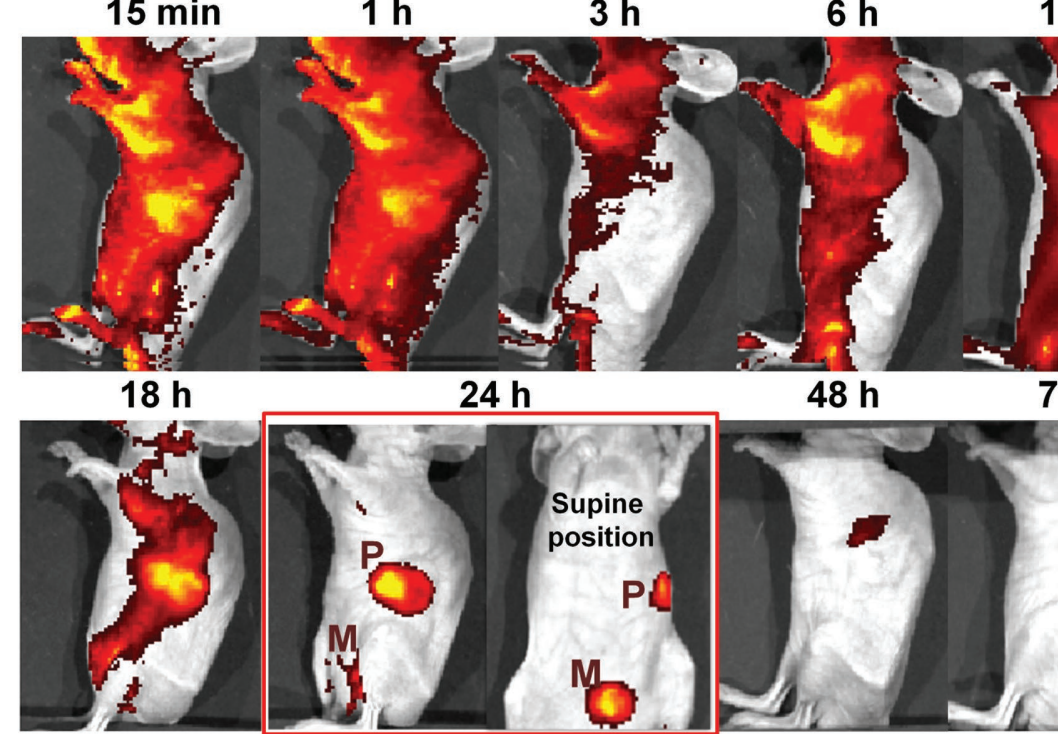

$12 \mathrm{~h} \mathrm{p} / \mathrm{sec} / \mathrm{cm}^{2} / \mathrm{sr}$

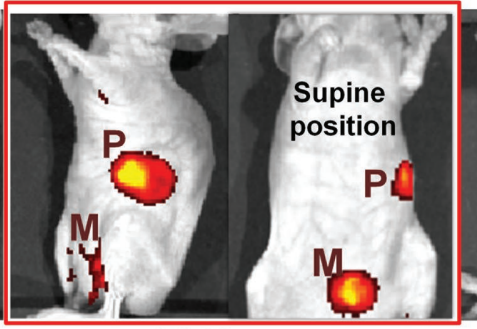

$48 \mathrm{~h}$
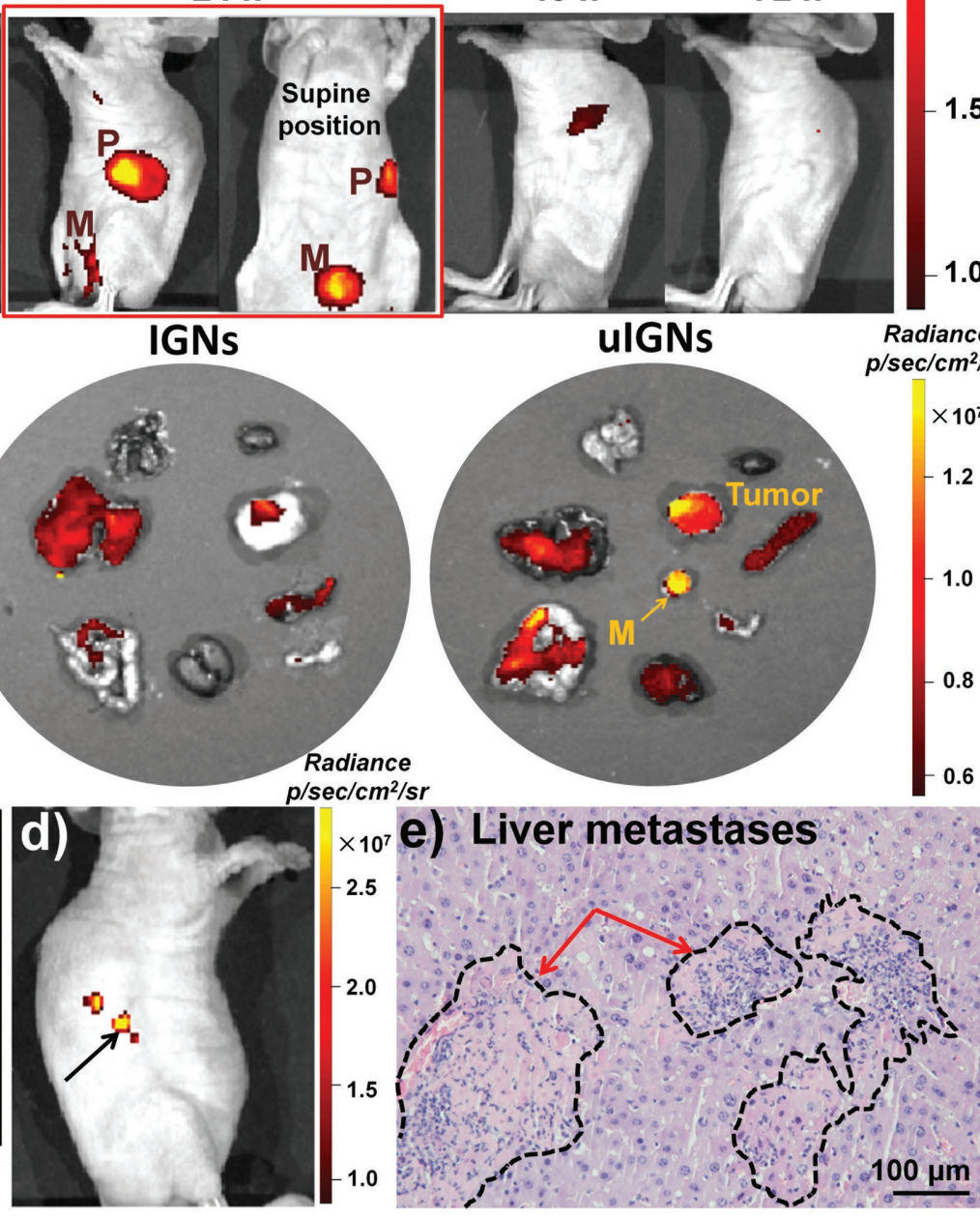

Figure 3. Biodistribution and fluorescence imaging. SW 1990 tumor-bearing mice were injected with ICG, IGNs and uIGNs $\left(250 \mu \mathrm{g} \mathrm{mL}^{-1}, 100 \mu \mathrm{L}\right)$ through the tail vein. a) In vivo continuous observations of the mice after administration of uIGNs using ICG fluorescence imaging. The primary tumor and peritoneal metastasis of the tumor-bearing mice were detected by BLI. P means primary pancreatic tumor. M means metastatic tumor. b) Ex vivo imaging of the major organs and tumor tissues at $24 \mathrm{~h}$ post-injection. Metastatic tumor is indicated by arrow. c) Comparison of T/N ratio profiles of tumor tissues based on ex vivo images. Data represent mean \pm SD of 3 replicate mice. d) Fluorescence imaging of multiple liver metastases (black arrow) post-injection of uICNs. e) H\&E staining of liver metastases circled by the dashed lines and indicated by red arrows. Scale bar is $100 \mu \mathrm{m}$.

groups (A-E) were 100\%, 37.5\%, 0\%, 0\%, and 0\%, respectively. Quantitative measurements indicated that the tumor weights in Group A were significantly lower than those in Group B $(P<0.001)$, as well as in the other three groups $(P<0.001)$ (Figure S12a, Supporting Information and Figure $5 \mathrm{~b}$ ). In Group D, a steady increase in tumor size but a slow decrease in fluorescence intensity was observed (Figure 5a). This might be a result of the slow action of the anti-tumor effect of ${ }^{125}$ I radioactive seeds, which were only able to kill a small number of tumor cells during the $15 \mathrm{~d}$ observation owing to the fast growth (i.e., short doubling time) of SW1990 tumors. To preliminarily evaluate the toxicity of all treatments, mouse body weights were monitored over the course of the $15 \mathrm{~d}$ experimental treatments. No significant difference in body weight was observed between 

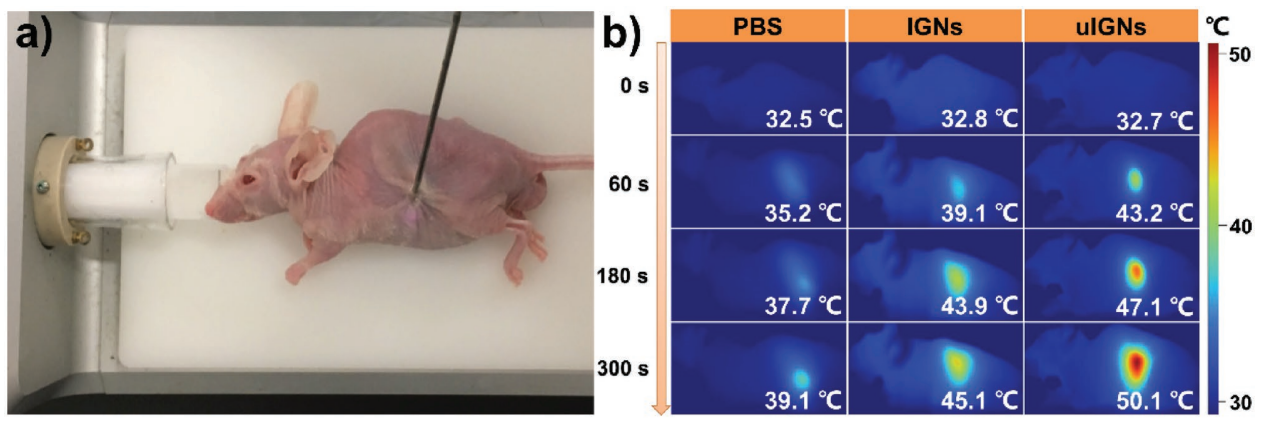

Figure 4. In vivo interventional photothermal therapy. a) The photo image of a tumor-bearing BALB/c nude mouse under an $808 \mathrm{~nm}$ NIR laser irradiation by a self-developed IPTT device. b) Infrared thermal images of the tumor-bearing mice at 24 h post-injection of PBS, IGNs, uIGNs via tail vein before and after NIR laser light irradiation $\left(2.0 \mathrm{~W} \mathrm{~cm}^{-2}, 300 \mathrm{~s}\right)$. The color bar relates the relative temperature values in ${ }^{\circ} \mathrm{C}$.

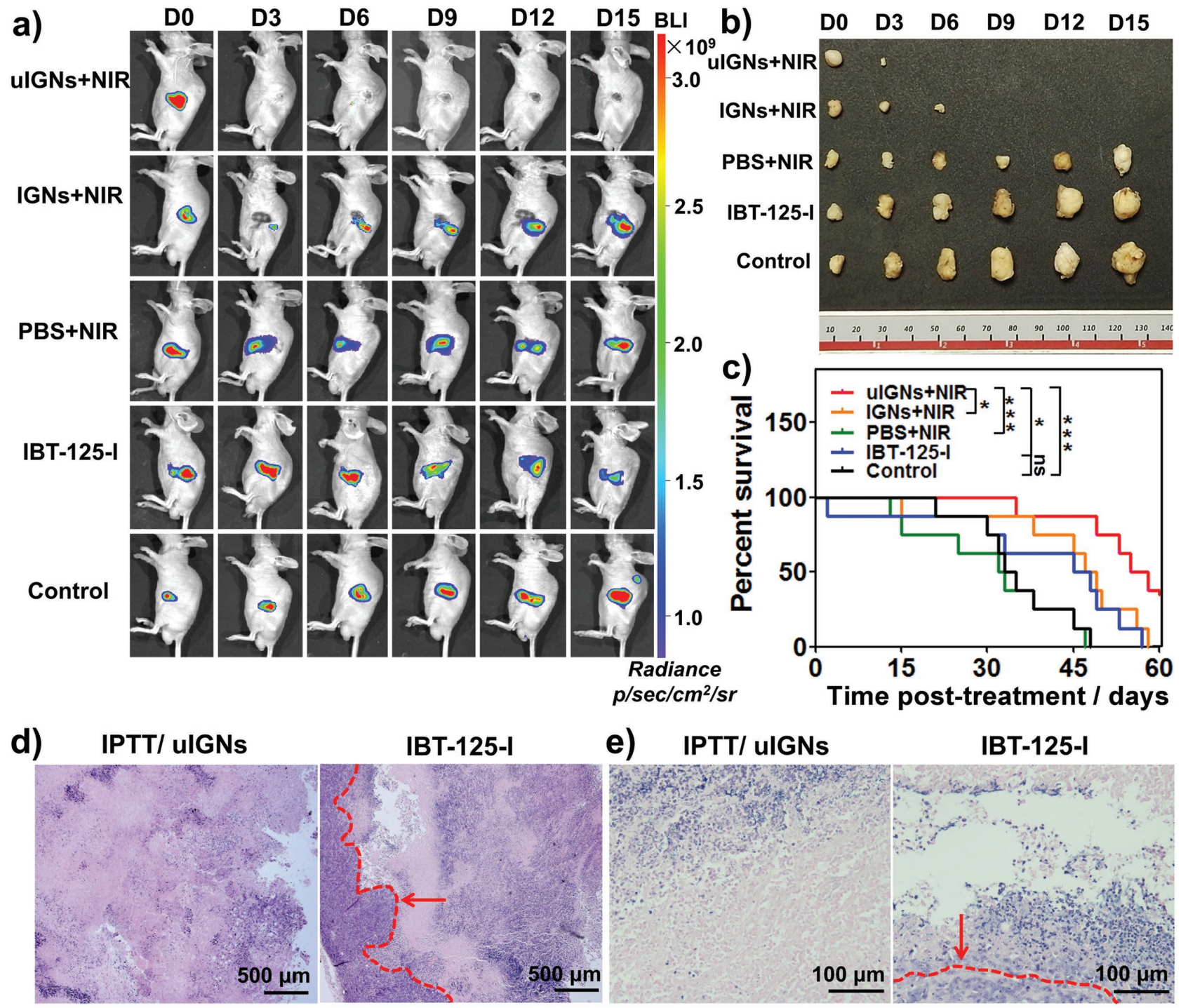

Figure 5. Evaluation of the therapeutic effects. a) BLI was utilized to continuously monitor the treated lesions at $3 \mathrm{~d}$ interval in different groups. b) Photo images of the resected tumors in different treatment groups at $3 \mathrm{~d}$ interval. c) The mice in different groups were monitored for $60 \mathrm{~d}$ following treatment and percent survival was calculated. d,e) Tumor tissues treated by uIGN-mediated IPTT and IBT-125-I were resected post-treatment and H\&E stain assay was performed. Large area of necrosis was observed in tumor tissues from both treatment groups, but residual live tumor cells were only observed in IBT-125-I treated tissues (circled by the dashed line and indicated by red arrow). Scale bar is $500 \mu \mathrm{m}$ in (d) and $100 \mu \mathrm{m}$ in (e). ns: $P>0.05 ; * P<0.05 ; * * * P<0.001$. 
IPTT-treated groups and the control group (Figure S12b, Supporting Information), suggesting that IPTT, to a certain extent, does not elicit acute or fatal systemic toxicity.

To investigate the prognosis and long-term survival rates resulting from IPTT, mice were monitored for $60 \mathrm{~d}$ following treatment. As shown in Figure $5 \mathrm{c}$, the average survival rate of the mice in Group A was significantly higher than that of the mice in Group B $(P<0.05)$, Group C $(P<0.001)$, Group D $(P<0.05)$, and Group E $(P<0.001)$. The median survival for the five treatment groups were $56.5,48.0,32.5,46.5$, and $34.0 \mathrm{~d}$, respectively. Most notably, the median survival rate of the uIGN group was prolonged by $66.2 \%$ and $25.0 \%$, compared to the control group and IBT-125-I group, respectively.

To further compare the anti-tumor efficiency of uIGN-mediated IPTT and IBT-125-I, hematoxylin and eosin (H\&E) staining was performed. Both treatments resulted in tremendous cellular damage of cancerous tissues, but not normal tissues; however, residual and undamaged tumor cells were detected in ${ }^{125} \mathrm{I}$ seed-treated tumor tissues (Figure 5d,e).

In this study, we also monitored the onset and progression of tumor metastases via BLI every $3 \mathrm{~d}$ post-treatment for $30 \mathrm{~d}$. As is well known, tumor metastasis is a multistep process involving migration and invasion from primary tumor lesion, intravasation into blood/lymphatic vessels and survival in the circulation system, extravasation into distant tissues, and establishment of metastatic foci in the seeded distant tissues. ${ }^{[17]}$ Effective suppression of tumor metastasis can be achieved by interrupting any of these steps. ${ }^{[18]}$ During the $30 \mathrm{~d}$ post-treatment observation period, tumor metastases occurred in 1 out of 8 mice in the PBS/laser group, in 3 out of 8 mice in the IBT-125-I group, and in 4 out of 8 mice in the control group. Interestingly, there were no metastases observed in uIGNs/laser- and IGNs/lasertreated groups (Table S1). While the mechanisms underlying this phenomenon are unclear, it could be the result of complete elimination of primary tumors or reduced tumor load after IPTT, inability to detect micrometastasis owing to the limitation of imaging modalities, or uncertain immune responses caused by PTT. ${ }^{[19]}$

In recent studies, several groups investigated the immune effects of PTT; although preliminary, the results of some of these studies are noteworthy. Wang et al. ${ }^{[20]}$ observed that the photothermal ablation of primary tumors with single-walled carbon nanotubes can trigger immune responses, and that this photothermal ablation can also inhibit tumor metastasis when used in combination with an anti-CTLA-4 antibody. Furthermore, Liang et al. ${ }^{[19 a]}$ observed that imaging-guided PTT with single-walled carbon nanotubes could effectively destroy primary tumors and metastatic cancer cells in sentinel lymph nodes, resulting in remarkably prolonged mouse survival, compared to surgical resection of the primary tumor alone. However, some researchers believe that the immune effect triggered by PTT is a double-edged sword. For example, Bear et al.[21] reported that PTT promotes the infiltration of secondary tumor sites by $\mathrm{CD} 11 \mathrm{~b}^{+} \mathrm{Ly}-6 \mathrm{G} / \mathrm{C}^{+}$myeloid-derived suppressor cells, thereby enhancing the growth of distant lung metastases of melanoma. Additionally, due to the lack of the architectural and cellular complexity and microenvironment of in vivo tumors, as well as a properly functioning immune system, tumor development and progression cannot be fully evaluated in immunodeficient xenografts. ${ }^{[18 b]}$ This existing problem and controversial result raise more questions about the mechanisms by which PTT induce immune responses. Therefore, to achieve optimal therapeutic effects while avoiding the adverse effects of PTT, it might be promising to explore new therapeutic strategies or combine PTT with other therapies such as immunotherapy, gene therapy, chemotherapy, etc. ${ }^{[18 b, 22]}$ Indeed, Mahajan et al. ${ }^{[18 b]}$ achieved remarkable results and proved that combined use of a targeting peptide and siRNAs could efficiently silence PLK1 and inhibit the growth of pancreatic tumors. However, substantial effort will be required to fully understand and exploit the functionalities of PTT and other treatments for effective cancer therapy.

These results demonstrate that under the conditions tested in this work, IPTT comprises a more safe and radical method for killing pancreatic tumor cells than IBT-125-I, and that IPTT-mediated method using targeted uIGNs are more effcient in eradicating PC than methods using non-targeted agents. Additionally, as reported by Skovgaard et al., ${ }^{[23]}$ an uPAR-targeting peptide labeled with $68 \mathrm{Ga}$ was evaluated in a phase-I clinical trial for Positron Emission Tomography (PET) imaging in breast, prostate, and urinary bladder cancers. The clinical evaluation of uIGNs component demonstrated the value and potential of UIGN-mediated IPTT in clinical translation.

In summary, the uIGNs prepared in this study demonstrated excellent tumor-targeting abilities and satisfactory biocompatibility. More importantly, we confirmed that an interventional method for uIGN-mediated PTT delivery could be utilized to treat PC, yielding a one-time successful treatment and prolonged survival period in mice. These findings imply that IPTT, with precise heating and reduced tissue injury, exhibits great potential for the effective treatment of deep-buried PC. As a result of the feasibility and effectiveness of this therapy, it is likely that this minimally invasive uIGN-mediated IPTT method could be translated into clinical practice in the near future, particularly for aged and/or high-risk patients, or for patients who develop metastases.

\section{Supporting Information}

Supporting Information is available from the Wiley Online Library or from the author.

\section{Acknowledgements}

This work was supported by the National Natural Science Foundation of China (grant number 51572271), (grant number 81471683), (grant number 81671710), (grant number 81227901), (grant number 81527805), (grant number 61501462), National Basic Research Program of China (973 Program) (grant number 2016YFA0201500), Fundamental Research Funds for the Central Universities (grant number buctrc201610), (grant number JD1609), (grant number PYBZ1705) and the Strategic Priority Research Program from Chinese Academy of Sciences (grant number XDB02060010). All animal experiments were approved by the Animal Ethics Committee of General Hospital of People's Liberation Army and were carried out in compliance with the Animal Management Rules of the Ministry of Health of the People's Republic of China (Document NO. 55,2001$)$. 


\section{Conflict of Interest}

The authors declare no conflict of interest.

\section{Keywords}

active targeting, clinical intervention, gold nanoshells, interventional photothermal therapy, pancreatic cancer

Received: January 22, 2017

Revised: April 15, 2017

Published online: July 6, 2017

[1] a) F. Puleo, R. Marechal, P. Demetter, M. A. Bali, A. Calomme, J. Closset, J. B. Bachet, J. Deviere, J. L. Van Laethem, World J. Gastroenterol. 2015, 21, 2281; b) A. Jemal, R. Siegel, J. Xu, E. Ward, CA Cancer J. Clin. 2010, 60, 277

[2] R. Spadi, F. Brusa, A. Ponzetti, I. Chiappino, N. Birocco, L. Ciuffreda, M. A. Satolli, World J. Clin. Oncol. 2016, 7, 27.

[3] a) Z. Jin, Y. Du, Z. Li, Y. Jiang, J. Chen, Y. Liu, Endoscopy 2008, 40, 314; b) H. Badakhshi, R. Graf, V. Budach, P. Wust, Strahlenther. Onkol. 2015, 191, 303.

[4] P. Huang, P. Rong, A. Jin, X. Yan, M. G. Zhang, J. Lin, H. Hu, Z. Wang, X. Yue, W. Li, G. Niu, W. Zeng, W. Wang, K. Zhou, X. Chen, Adv. Mater. 2014, 26, 6401

[5] a) S. Wang, A. Riedinger, H. Li, C. Fu, H. Liu, L. Li, T. Liu, L. Tan, M. J. Barthel, G. Pugliese, F. De Donato, M. Scotto D'Abbusco, X. Meng, L. Manna, H. Meng, T. Pellegrino, ACS Nano 2015, 9, 1788; b) P. Vijayaraghavan, C. H. Liu, R. Vankayala, C. S. Chiang, K. C. Hwang, Adv. Mater. 2014, 26, 6689.

[6] X. Qian, Y. Zheng, Y. Chen, Adv. Mater. 2016, 28, 8097.

[7] A. D. Fesnak, C. H. June, B. L. Levine, Nat. Rev. Cancer 2016, 16, 566.

[8] a) S. Akhter, M. Z. Ahmad, F. J. Ahmad, G. Storm, R. J. Kok, Expert Opin. Drug Delivery 2012, 9, 1225; b) F. Kong, H. Zhang, X. Qu, X. Zhang, D. Chen, R. Ding, E. Makila, J. Salonen, H. A. Santos, M. Hai, Adv. Mater. 2016, 28, 10195.

[9] a) H. Liu, D. Chen, L. Li, T. Liu, L. Tan, X. Wu, F. Tang, Angew. Chem., Int. Ed. 2011, 50, 891; b) H. Liu, T. Liu, L. Li, N. Hao, L. Tan, X. Meng, J. Ren, D. Chen, F. Tang, Nanoscale 2012, 4, 3523; c) H. Liu, T. Liu, H. Wang, L. Li, L. Tan, C. Fu, G. Nie, D. Chen, F. Tang, Biomaterials 2013, 34, 6967; d) H. Liu, D. Chen, F. Tang, G. Du, L. Li, X. Meng, W. Liang, Y. Zhang, X. Teng, Y. Li, Nanotechnology 2008, 19, 455101; e) H. Liu, T. Liu, X. Wu, L. Li, L. Tan, D. Chen, F. Tang, Adv. Mater. 2012, 24, 755.

[10] M. F. Tsai, S. H. Chang, F. Y. Cheng, V. Shanmugam, Y. S. Cheng, C. H. Su, C. S. Yeh, ACS Nano 2013, 7, 5330.
[11] a) W. Chen, S. Zhang, Y. Yu, H. Zhang, Q. He, Adv. Mater. 2016, 28, 8567; b) R. Bardhan, S. Lal, A. Joshi, N. J. Halas, Acc. Chem. Res. 2011, 44, 936; c) S. Lal, S. E. Clare, N. J. Halas, Acc. Chem. Res. 2008, 41, 1842.

[12] a) F. Blasi, N. Sidenius, FEBS Lett. 2010, 584, 1923; b) X. Tan, H. Egami, F. Nozawa, M. Abe, H. Baba, Int. J. Oncol. 2006, 28, 369; c) Y. Chen, B. Zheng, D. H. Robbins, D. N. Lewin, K. Mikhitarian, A. Graham, L. Rumpp, T. Glenn, W. E. Gillanders, D. J. Cole, X. Lu, B. J. Hoffman, M. Mitas, Int. J. Cancer 2007, 120, 1511.

[13] M. Erkan, S. Hausmann, C. W. Michalski, A. A. Fingerle, M. Dobritz, J. Kleeff, H. Friess, Nat. Rev. Gastroenterol. Hepatol. 2012, 9, 454.

[14] C. Zeng, W. Shang, X. Liang, X. Liang, Q. Chen, C. Chi, Y. Du, C. Fang, J. Tian, ACS Appl. Mater. Interfaces 2016, 8, 29232.

[15] S. Wang, P. Huang, X. Chen, Adv. Mater. 2016, 28, 7340.

[16] a) L. E. Cole, T. Vargo-Gogola, R. K. Roeder, ACS Nano 2015 9, 8923; b) J. Park, J. Park, E. J. Ju, S. S. Park, J. Choi, J. H. Lee, K. J. Lee, S. H. Shin, E. J. Ko, I. Park, C. Kim, J. J. Hwang, J. S. Lee, S. Y. Song, S. Y. Jeong, E. K. Choi, J. Controlled Release 2015, 207, 77; c) H. Deng, Y. Zhong, M. Du, Q. Liu, Z. Fan, F. Dai, X. Zhang, Theranostics 2014, 4, 904

[17] a) L. Wan, K. Pantel, Y. Kang, Nat. Med. 2013, 19, 1450; b) Q. He, S. Guo, Z. Qian, X. Chen, Chem. Soc. Rev. 2015, 44, 6258.

[18] a) A. Schroeder, D. A. Heller, M. M. Winslow, J. E. Dahlman, G. W. Pratt, R. Langer, T. Jacks, D. G. Anderson, Nat. Rev. Cancer 2011, 12, 39; b) U. M. Mahajan, S. Teller, M. Sendler, R. Palankar, C. van den Brandt, T. Schwaiger, J. P. Kuhn, S. Ribback, G. Glockl, M. Evert, W. Weitschies, N. Hosten, F. Dombrowski, M. Delcea, F. U. Weiss, M. M. Lerch, J. Mayerle, Gut 2016, 65, 1838

[19] a) C. Liang, S. Diao, C. Wang, H. Gong, T. Liu, G. Hong, X. Shi, H. Dai, Z. Liu, Adv. Mater. 2014, 26, 5646; b) J. Conde, N. Oliva, Y. Zhang, N. Artzi, Nat. Mater. 2016, 15, 1128.

[20] C. Wang, L. Xu, C. Liang, J. Xiang, R. Peng, Z. Liu, Adv. Mater. 2014, 26, 8154 .

[21] A. S. Bear, L. C. Kennedy, J. K. Young, S. K. Perna, J. P. Mattos Almeida, A. Y. Lin, P. C. Eckels, R. A. Drezek, A. E. Foster, PLoS One 2013, 8, e69073.

[22] a) L. Zou, H. Wang, B. He, L. Zeng, T. Tan, H. Cao, X. He, Z. Zhang, S. Guo, Y. Li, Theranostics 2016, 6, 762; b) Q. Chen, L. Xu, C. Liang, C. Wang, R. Peng, Z. Liu, Nat. Commun. 2016, 7, 13193; c) I. H. Lee, H. K. Kwon, S. An, D. Kim, S. Kim, M. K. Yu, J. H. Lee, T. S. Lee, S. H. Im, S. Jon, Angew. Chem., Int. Ed. 2012, 51, 8800; d) K. Niikura, T. Matsunaga, T. Suzuki, S. Kobayashi, H. Yamaguchi, Y. Orba, A. Kawaguchi, H. Hasegawa, K. Kajino, T. Ninomiya, K. Ijiro, H. Sawa, ACS Nano 2013, 7, 3926; e) R. Arvizo, R. Bhattacharya, P. Mukherjee, Expert Opin. Drug Delivery 2010, 7, 753.

[23] D. Skovgaard, M. Persson, M. Brandt-Larsen, C. Christensen, J. Madsen, T. L. Klausen, S. Holm, F. L. Andersen, A. Loft, A. K. Berthelsen, H. Pappot, K. Brasso, N. Kroman, L. Hoejgaard, A. Kjaer, J. Nucl. Med. 2017, 58, 379. 